\title{
Atypical juvenile parkinsonism
}

INSERM

\section{Source}

INSERM. (1999). Orphanet: an online rare disease and orphan drug data base. Atypical juvenile parkinsonism. ORPHA:391411

Atypical juvenile parkinsonism (AJP) is a complex form of young-onset Parkinson disease (YOPD; see this term) that manifests with pyramidal signs, eye movement abnormalities, psychiatric manifestations (depression, anxiety, drug-induced psychosis, and impulse control disorders), intellectual disability, and other neurological symptoms (such as ataxia and epilepsy) along with classical parkinsonian symptoms. 